\title{
Eventos potencialmente traumáticos y sintomatología postraumática asociada con el consumo de alcohol en hombres y mujeres estudiantes de bachillerato
}

\author{
Sheila Adriana Mendoza Mojica', Midiam Moreno López², Luciana Ramos Lira² \\ I Centro de Investigación en Ciencias Médicas, Universidad Autónoma del Estado de México, México \\ 2 Dirección de Investigaciones Epidemiológicas y Psicosociales, Instituto Nacional de Psiquiatría Ramón de la Fuente Muñiz, México
}

RESUMEN

Introducción: los eventos adversos pueden afectar la salud mental y considerarse "potencialmente traumáticos". El uso de alcohol puede configurarse como estrategia de "automedicación" para abordar la sintomatología postraumática asociada. La victimización, al igual que su impacto, puede mostrar un perfil diferente por el tipo de evento y el sexo de la persona afectada. Objetivo: explorar la asociación entre la exposición a eventos potencialmente traumáticos, la sintomatología postraumática y el consumo de alcohol en estudiantes de bachillerato de ambos sexos. Método: se realizó un estudio analítico de corte transversal en una muestra de mil estudiantes de bachillerato. Se empleó la Escala para Estrés Postraumático en Universitarios Mexicanos (EEPUM) y un cuestionario para detectar el abuso de alcohol. Se analizó, asimismo, la asociación entre los eventos potencialmente traumáticos y los síntomas postraumáticos con el consumo de alcohol. Resultados: cuatro de cada cinco participantes experimentaron un posible evento postraumático; una proporción similar consideró un evento con afectación actual. Se observaron altos porcentajes de sintomatología postraumática: al menos un síntoma en más de $80 \%$ de las personas. El consumo de alcohol fue más frecuente entre las mujeres que experimentaron un evento posiblemente traumático-específicamente violencia interpersonal-, en comparación con las que no lo vivieron. En hombres, el consumo fue similar entre los que experimentaron un evento adverso y los que no. Discusión y conclusiones: la adversidad y la violencia son una realidad diaria para los jóvenes, y estas situaciones tienen un gran impacto en su bienestar y salud mental. Los eventos posiblemente traumáticos se asociaron con el consumo de alcohol sólo en mujeres, por lo que estos hallazgos deberían profundizarse, incluso si la violencia interpersonal es la más asociada con este consumo.

Palabras claves: eventos traumáticos, Trastorno por Estrés Postraumático, abuso de alcohol, estudiantes.
ABSTRACT

Introduction: adverse events can affect the mental health of those who suffer them, so they can be called "potentially traumatic". Among these effects, the use of alcohol is very important, since it can be configured as a "self-medication" strategy to address the associated post-traumatic symptomatology. Victimization, like its impact, can show a different profile of the type of event and sex. Objective: to explore the association between exposure to potentially traumatic events, post-traumatic symptomatology and alcohol consumption in high school students of both sexes. Method: a cross-sectional analytical study was carried out on a sample for the convenience of a thousand students from three high schools. The Post-Traumatic Stress Scale was applied to Mexican university students and an alcohol abuse scale. The association between potentially traumatic events and post-traumatic symptoms with alcohol consumption was analyzed. Results: four out of five participants experienced a possible post-traumatic event; a similar proportion had an event that affected them until today. High percentages of post-traumatic symptomatology were observed: at least one symptom in more than $80 \%$ of the participants. Alcohol consumption was more frequent among women who experienced a possibly traumatic event, specifically interpersonal violence, compared to those who did not. In men, consumption was similar between those who experienced an adverse event and those who did not. Discussion and conclusions: adversity and violence are a daily reality for young people, and these situations have a great impact on their well-being and mental health. The possibly traumatic events were associated with alcohol consumption only in women, so these findings should be deepened, even if interpersonal violence is the most associated with this consumption.

Keywords: traumatic events, Post-Traumatic Stress Disorder, alcohol abuse, students.

\footnotetext{
Autor de correspondencia:

Sheila Adriana Mendoza Mojica. Jesús Carranza núm. 205, col. Universidad, Toluca, C.P. 50130. Estado de México, México. Correo electrónico: shememo06@yahoo.com.mx

Recibido: 11 de abril de 2018

Aceptado: 07 de mayo de 2018

doi:10.28931/riiad.2018.1.3
} 


\section{INTRODUCCIÓN}

De acuerdo con la Encuesta de Cohesión Social para la Prevención de la Violencia y la Delincuencia (ECOPRED, 2014), la violencia es uno de los problemas sociales más graves que afectan a la población mexicana, en particular a la más joven (Instituto Nacional de Estadística y Geografía, 2015). En prácticamente todo el mundo, los hombres adolescentes son victimizados, con más frecuencia que las mujeres, por actos de violencia física en los espacios públicos (Löfving-Gupta et al., 2017), mientras que éstas padecen más violencia sexual tanto dentro como fuera de sus hogares, a cualquier edad (Organización Mundial de la Salud [OMS], 2013).

Se han documentado diversos efectos en la salud mental asociados con la exposición a la violencia (OMS, 2002), los más graves son los que se producen por eventos ocurridos en edades tempranas (Teicher \& Samson, 2016), los que surgen por experimentar múltiples tipos de victimización (Hughes et al., 2017) o por estar expuesto a violencia crónica y de larga duración (Kaysen et al., 2007). Además de la depresión y el Trastorno por Estrés Postraumático (TEPT), se ha observado un aumento de problemas con el consumo de alcohol (Kaysen et al., 2007).

Las experiencias adversas de la infancia (como los abusos físico, sexual, emocional, psicológico o verbal y la exposición a la violencia doméstica) son factores de riesgo para la mayoría de las categorías de trastorno mental: trastornos del estado de ánimo, de ansiedad, del comportamiento y de uso de sustancias a lo largo de la vida (Kessler et al., 2010). Sin embargo, el impacto de la victimización violenta varía según el sexo y el tipo de trauma; una revisión sistemática reciente de 72 artículos sobre TEPT en niños, niñas y adolescentes (Alisic et al., 2014) muestra que $15.9 \%$ de las personas lo desarrolla tras un suceso traumático, pero los varones presentan un porcentaje menor de TEPT (8.4\%), que se asocia, principalmente, con sucesos traumáticos no interpersonales como accidentes. Las niñas, en contraste, reportan el padecimiento cuatro veces más (32.9\%) y se asocia con sucesos interpersonales. La victimización sexual es un predictor significativo de TEPT y de comportamientos agresivos en las niñas (Cyr et al., 2017); de hecho, es el tipo de violencia que tiene más probabilidad de generar un TEPT (Medina-Mora et al., 2005; Ramos-Lira, Saltijeral-Méndez, Romero-Mendoza, Caballero-Gutiérrez, \& Martínez Vélez, 2001).

En este sentido, ciertos sucesos violentos pueden tener un efecto importante en el consumo de alcohol de los y las adolescentes, entre ellos se enumeran el abuso sexual infantil (Hughes et al., 2017), el mal- trato físico y emocional (Hill, Shen, Lowers, \& Locke, 2000), la violencia comunitaria (Fairbrook, 2013; Löfving-Gupta et al., 2017; Mels \& Fernández, 2015) y la que se da en el noviazgo (Shorey, Rhatigan, Fite, \& Stuart, 2011).

En su revisión sistemática y metaanálisis, Hughes et al. (2017) encontraron que las personas que habían experimentado al menos cuatro experiencias adversas en la infancia (como exposición a la violencia doméstica, o abuso físico, sexual, emocional, psicológico o verbal) tenían más del doble de probabilidad de ser fumadoras actuales o bebedoras fuertes, en comparación con quienes no habían tenido ninguna experiencia; además, eran casi seis veces más propensas a beber problemáticamente.

Lo anterior ha llevado a sugerir que el consumo excesivo de alcohol en adolescentes puede ser una forma de afrontar los efectos negativos de la exposición a la violencia (Kilpatrick et al., 2000). En este caso, el alcohol se usa como una automedicación para reducir las dificultades del sueño, el afecto negativo, la tensión y los síntomas intrusivos y de hiperactivación, característicos del TEPT. Así, quienes tienen síntomas postraumáticos graves y creen que el alcohol es una forma útil para sobrellevarlos, corren un mayor riesgo de consumo excesivo y de presentar conductas desadaptativas; también puede ocurrir que exista una relación directa entre la gravedad de los síntomas postraumáticos y la forma de beber alcohol (Kaysen et al., 2007).

Orozco, Borges, Benjet, Medina-Mora y López-CarriIlo (2008) encontraron que $68.9 \%$ de los adolescentes de 12 a 17 años residentes en el Área Metropolitana de la Ciudad de México había experimentado un estresor traumático alguna vez en su vida. Por su parte, Gómez Hernández y Gómez-Maqueo (2016), al hacer un estudio con estudiantes, reportaron que $81.7 \%$ de los adolescentes encuestados había estado expuesto de manera directa a por lo menos un suceso violento en su comunidad; $92.7 \%$ dijo haber sido testigo de al menos uno. De manera global, $96.6 \%$ de los alumnos aceptó haber estado expuesto a un evento violento, ya sea de manera directa o indirecta.

El estudio de Mendoza-Mojica, Márquez-Mendoza, Veytia-López, Ramos-Lira y Orozco-Zavala (2017) en estudiantes de bachillerato mostró que $80 \%$ había experimentado al menos un evento potencialmente traumático; en promedio, los participantes reportaron 2.95 eventos de este tipo que ocurrieron alrededor de los 13 años. Prácticamente todos los estudiantes (97\%) que experimentaron un evento con repercusión actual mostraron al menos un síntoma de estrés postraumático, en tanto que $45 \%$ reportó al menos uno en todos los factores. 
Con base en lo anterior, consideramos importante explorar en esta población, la asociación entre la exposición a eventos potencialmente traumáticos y un mayor consumo de alcohol; el presente trabajo tiene como objetivo identificar si existen diferencias entre hombres y mujeres estudiantes de nivel bachillerato, en cuanto a la exposición a eventos potencialmente traumáticos, tipo de evento, sintomatología postraumática y consumo de alcohol.

\section{MÉTODO}

\section{Diseño del estudio}

Se hizo un estudio transversal analítico que tuvo como objetivo explorar las diferencias entre hombres y mujeres estudiantes de nivel bachillerato, respecto a la exposición a Eventos Potencialmente Traumáticos (EVPT), tipo de evento, repercusión actual, sintomatología postraumática y consumo de alcohol.

\section{Sujetos}

Se eligieron por conveniencia tres escuelas de bachillerato ubicadas en el Estado de México, específicamente en los siguientes municipios: Luvianos (al sur del estado, colinda con Michoacán y Guerrero), donde desde el año 2009 se han suscitado desapariciones y situaciones de violencia, como resultado de la Guerra contra el Narcotráfico; Tlatlaya (también al sur del Estado de México), que cuenta con la mayor extensión territorial de la entidad, y donde, en 2014, asesinaron extrajudicialmente a 22 personas que supuestamente eran parte de un grupo delictivo (Ferri, 2014; Instituto Nacional para el Federalismo y Desarrollo Municipal [INAFED], 2017; Proceso, 2011; Proceso, 2014); y Ecatepec (al norte del estado y del Valle de México, colinda con la Ciudad de México y se considera parte de su zona metropolitana), municipio en el que la población afronta diversos desastres naturales y causados por el ser humano, y donde los delitos denunciados sólo representan $8 \%$ del total de los que ocurren, principalmente abuso sexual y físico (Cabildo, 2011; INAFED, 2017).

De 1,436 hombres y mujeres inscritos durante el periodo 2015-2016, se consideró una muestra de 1,000 estudiantes (436 hombres y 564 mujeres) que aceptaron participar voluntariamente en el estudio. El promedio de edad fue de 16.18 años; $23.6 \%$ de los participantes era de Luvianos, $26.3 \%$ de Tlatlaya y $50.1 \%$ de Ecatepec. De ellos, 634 eligieron de la lista un EVPT con impacto en la actualidad, lo describieron brevemente y respondieron la escala de síntomas de TEPT subumbral durante el último año.

\section{Mediciones}

La frecuencia de EVPT experimentado alguna vez en la vida y la sintomatología de TEPT subumbral en el último año fueron indagadas mediante la Escala para Estrés Postraumático en Universitarios Mexicanos (EEPUM), un instrumento autoadministrado que solicita datos sociodemográficos (sexo, edad y municipio). En su primera sección, consta de una lista de 31 EVPT (además de "otro, especifique"), en la que los participantes marcan qué eventos han vivido; al final, se pide lo siguiente a quienes dijeron haber vivido al menos un EVPT: "Escoge el suceso que consideres más impactante y que aún afecta tu bienestar y seguridad" (Mendoza-Mojica, Márquez, Guadarrama, \& Ramos, 2013).

La escala se compone de cinco factores y, como instrumento de tamizaje, incluye los síntomas de los criterios B-E del DSM-5: 1) evitación de tipo embotamiento emocional, 2) reexperimentación de tipo fisiológica, 3) evitación de tipo alejamiento, 4) hiperactivación y 5) reexperimentación de tipo cognitiva, con valores de confiabilidad interna que van desde $a=.71$ (factor 4 ) hasta $a=.96$ (factor 1), pero que no considera los criterios $F$ (duración), G (malestar o deterioro) o H (exclusiones orgánicas) del DSM-5 (American Psychiatric Association [APA], 2013). Estos valores psicométricos concuerdan con los reportados de manera previa en población universitaria $(N=669)$, posee una confiabilidad interna $a=.95$ general y valores de $a=.70$ (factor 4 ) hasta $a=.91$ (factor 1); la varianza explicada fue de $45 \%$ general (Mendoza-Mojica et al., 2013). El uso del instrumento sólo permite detectar síntomas de TEPT subumbral, sin precisar el diagnóstico completo, mediante las opciones de respuestas: $0=$ nada, $1=$ algunas veces, 2 = una vez al mes, 3 = una vez a la semana y $4=$ diariamente (Mendoza-Mojica et al., 2017).

El nivel de consumo de alcohol se obtuvo con un cuestionario de nueve preguntas basado en la Escala de Involucramiento con el Alcohol en Adolescentes de Mayer y Filstead (1979, citado por Jiménez, 2004). Cada pregunta contó con opciones de respuesta específicas que permitieron identificar, además de la frecuencia de uso de la sustancia, los efectos del alcohol en el funcionamiento psicológico, así como algunos aspectos de las relaciones sociales y familiares en torno al consumo.

\section{Procedimientos}

Una vez autorizada la aplicación del instrumento por parte de la autoridad escolar, se coordinó a los orientadores educativos, para que una semana antes, les explicaran a los estudiantes su participación en el estudio, mediante la lectura del consentimiento y asentimiento informados. 
Se les pidió que lo completaran con sus padres y entregaran el día de la aplicación. El tiempo para responder los instrumentos fue de aproximadamente una hora; la aplicación fue grupal y estuvo a cargo de dos psicólogos previamente capacitados, quienes se aseguraron de que los estudiantes participaran de manera voluntaria en el estudio y preservaron el manejo confidencial de los datos obtenidos.

\section{Análisis estadísticos}

Las variables relacionadas con la experimentación de EVPT fueron dicotómicas (sí/no); se obtuvo el porcentaje de los participantes que vivieron algún evento y manifestaron tener alguna repercusión actual. Los 31 eventos se agruparon en cinco tipos: 1. Exposición y participación en la violencia organizada; 2 . Violencia interpersonal; 3. Violencia sexual; 4. Otras amenazas para la vida; 5. Experiencias traumáticas en red. Las variables relacionadas con la presencia de sintomatología de TEPT subumbral fueron dicotómicas y por factor de la EEPUM. Para las preguntas del consumo de alcohol se obtuvieron las frecuencias de ocurrencia de respuesta. Las diferencias estadísticas entre las variables sobre consumo de alcohol y los eventos, de acuerdo con el sexo del participante, se obtuvieron mediante la obtención de intervalos de confianza a 95\%. El procesamiento y análisis de datos se hizo a nivel exploratorio utilizando el paquete estadístico STATA versión 13 (StataCorp, Texas, E.U.); los intervalos de confianza se obtuvieron mediante el comando proportion.

El consentimiento y asentimiento informados fueron elaborados con base en las consideraciones del Tratado de Helsinki para estudios con sujetos; asimismo, fueron sometidos y aprobados, al igual que el protocolo del estudio, al Comité de Investigación y Ética del Centro de Investigación en Ciencias Médicas de la Universidad Autónoma del Estado de México.

\section{RESULTADOS}

En la Tabla 1 se aprecia que del total de los 1,000 estudiantes participantes, $80.2 \%$ afirmó haber experimentado un evento potencialmente traumático. De estos, $79.1 \%$ dijo que éste seguía teniendo una repercusión en la actualidad. Se observa, también, que los eventos de violencia interpersonal fueron experimentados por $53.1 \%$, mientras que las experiencias traumáticas en red fueron mencionadas por $57.9 \%$.

634 reportaron sintomatología de TEPT subumbral en el último año, relacionada con el evento elegido. De ellos, 87.4\% manifestó tener síntomas de hiperactivación y $60.7 \%$, reexperimentación de tipo fisiológica en la actualidad. En la sección referente al consumo de alcohol, 151 jóvenes dejaron incompleto el cuestionario; del resto, $73.9 \%$ dijo haber usado la sustancia alguna vez en su vida.

Tabla 1

Descripción de las variables en el total de estudiantes de bachillerato

\begin{tabular}{|c|c|c|c|c|}
\hline \multirow[t]{2}{*}{ Variables } & \multicolumn{2}{|c|}{ No } & \multicolumn{2}{|c|}{ Sí } \\
\hline & $n$ & $\%$ & $n$ & $\%$ \\
\hline Vivió algún evento & 198 & 19.8 & 802 & 80.2 \\
\hline Exposición y participación en la violencia organizada & 520 & 64.8 & 282 & 35.2 \\
\hline Violencia interpersonal & 376 & 46.9 & 426 & 53.1 \\
\hline Violencia sexual & 753 & 93.9 & 49 & 6.1 \\
\hline Otras amenazas para la vida & 215 & 26.8 & 587 & 73.2 \\
\hline Experiencias traumáticas en red & 338 & 42.1 & 464 & 57.9 \\
\hline Consideraron un evento con repercusión actual & 168 & 20.9 & 634 & 79.1 \\
\hline Síntomas de evitación de tipo embotamiento emocional & 110 & 17.4 & 524 & 82.6 \\
\hline Síntomas de reexperimentación de tipo fisiológica & 249 & 39.3 & 385 & 60.7 \\
\hline Síntomas de evitación de tipo alejamiento & 108 & 17.0 & 526 & 83.0 \\
\hline Síntomas de hiperactivación & 80 & 12.6 & 554 & 87.4 \\
\hline Ha tomado alcohol alguna vez en su vida & 222 & 26.1 & 627 & 73.9 \\
\hline
\end{tabular}

Notas:

a) De los 1,000 estudiantes participantes, 802 vivieron de uno a 17 eventos de vida de distintos tipos; 634 manifestaron repercusión actual con variación en el factor de sintomatología.

b) 151 del total de participantes no respondieron el cuestionario de consumo de alcohol. 
En la Tabla 2 se observa la frecuencia de las características distintivas del consumo de alcohol por sexo. Por lo general, la primera causa por la que hombres y mujeres empiezan a tomar es porque "les gusta el sabor"; la segunda es "por problemas emocionales" y, finalmente, por "parecerse a sus amigos". La mayoría de los hombres $(55.3 \%)$ y mujeres $(56.3 \%)$ empezaron a consumir alcohol entre los 10 y los 15 años. Respecto a la forma de obtener las bebidas alcohólicas, se encontraron diferencias por sexo: $47.4 \%$ [IC $=42.2,52.7]$ de las mujeres reportó que las consiguen principalmente de sus "padres, hermanos u otros familiares"; en tanto que 30.3\% de los hombres afirmó conseguirlas de la misma fuente $[I C=25.1,36.0]$.

Tres cuartas partes de hombres $(76.9 \%)$ y mujeres $(77.8 \%)$ señalaron que la primera vez que tomaron bebidas alcohólicas lo hicieron por curiosidad. Sobre el número de copas que consumieron, hubo diferencias por sexo: $53.4 \%$ de las mujeres consumen de una a dos copas [IC $=48.2,58.6]$, frente a $37.1 \%$ de los hombres
[IC = 31.6, 43.0]; en este sentido, resaltó que mientras $16.4 \%$ de los hombres consume hasta emborracharse [IC $=12.4,21.2]$, sólo $6.3 \%$ de las mujeres lo hace así $[I C=4.1,9.3]$.

De la misma manera, más mujeres [45.2\%, IC $=40.0$, 50.4] que hombres [29.1\%, IC $=24.0,34.8$ ] reportaron que toman alcohol con sus padres, hermanos o familiares; y a la inversa, más hombres $[61.5 \%, I C=55.5,67.1]$ que mujeres $[48.9 \%, I C=43.6,54.1]$ mencionaron que consumen con sus amigos.

Respecto al efecto mayor que reportaron los jóvenes sobre su consumo de alcohol, cabe resaltar que $11.6 \%$ de hombres y $7.7 \%$ de mujeres mencionaron que se sintieron enfermos, se desmayaron o al día siguiente no se acordaban de lo que había pasado. Asimismo, 10.7\% reportó que su familia o amigos le aconsejan que controle o reduzca su consumo y $2.7 \%$ mencionó que su familia y amigos le han buscado ayuda para dejar de beber. A pesar de esto, casi la mitad de los participantes (51.2\%) dijo no tener problemas con su consumo de alcohol.

Tabla 2

Características distintivas del consumo de alcohol entre hombres y mujeres

\begin{tabular}{|c|c|c|c|c|c|c|c|c|c|}
\hline \multirow[b]{2}{*}{ Variables } & \multicolumn{3}{|c|}{ Hombre } & \multicolumn{3}{|c|}{ Mujer } & \multicolumn{3}{|c|}{ Total } \\
\hline & $n$ & $\%$ & IC 95\% & $n$ & $\%$ & IC 95\% & $n$ & $\%$ & IC 95\% \\
\hline \multicolumn{10}{|l|}{ Empiezo a beber porque } \\
\hline Me gusta el sabor & 191 & $71.3^{*}$ & {$[65.5,76.4]$} & 236 & $67.8^{*}$ & {$[62.7,72.5]$} & 427 & $69.3^{*}$ & {$[65.6,72.8]$} \\
\hline Para parecerme a mis amigos & 27 & $10.1^{*}$ & {$[7.0,14.3]$} & 21 & $6.0^{*}$ & {$[4.0,9.1]$} & 48 & $7.8^{*}$ & {$[5.9,10.2]$} \\
\hline Por problemas emocionales & 50 & $18.7^{\star}$ & {$[14.4,23.8]$} & 91 & $26.1^{*}$ & {$[21.8,31.0]$} & 141 & $22.9^{*}$ & {$[19.7,26.4]$} \\
\hline \multicolumn{10}{|l|}{ Edad de inicio de alcohol } \\
\hline Antes de los 10 años & 28 & $10.2^{*}$ & {$[7.1,14.4]$} & 16 & $4.5^{\star}$ & {$[2.8,7.3]$} & 44 & $7.0^{*}$ & {$[5.3,9.3]$} \\
\hline Entre los 10 y 15 años & 152 & $55.3^{*}$ & {$[49.3,61.1]$} & 198 & $56.3^{*}$ & {$[51.0,61.4]$} & 350 & $55.8^{*}$ & {$[51.9,59.7]$} \\
\hline Después de los 15 años & 95 & $34.5^{\star}$ & {$[29.1,40.4]$} & 138 & $39.2^{*}$ & {$[34.2,44.4]$} & 233 & $37.2^{*}$ & {$[33.5,41.0]$} \\
\hline \multicolumn{10}{|l|}{ Cómo obtiene sus bebidas } \\
\hline De padres, hermanos o familiares & 83 & $30.3^{\star *}$ & {$[25.1,36.0]$} & 167 & $47.4^{\star \star}$ & {$[42.2,52.7]$} & 250 & 39.9 & {$[36.2,43.8]$} \\
\hline De amigos & 94 & 34.3 & {$[28.9,40.2]$} & 127 & 36.1 & {$[31.2,41.3]$} & 221 & 35.3 & {$[31.6,39.1]$} \\
\hline Las consigo por mi cuenta & 97 & 35.4 & {$[29.9,41.3]$} & 58 & 16.5 & {$[12.9,20.7]$} & 155 & 24.8 & {$[21.5,28.3]$} \\
\hline \multicolumn{10}{|c|}{ ¿Por qué tomaste bebidas alcohólicas por primera vez? } \\
\hline Por curiosidad & 210 & $76.9^{\star}$ & {$[71.5,81.6]$} & 273 & $77.8^{*}$ & {$[73.1,81.8]$} & 483 & 77.4 & {$[73.9,80.5]$} \\
\hline Me la ofrecieron mis padres o familiares & 24 & 8.8 & {$[5.9,12.8]$} & 35 & 10.0 & {$[7.2,13.6]$} & 59 & 9.5 & {$[7.4,12.0]$} \\
\hline Mis amigos me animaron a beber & 29 & $10.6^{*}$ & {$[7.5,14.9]$} & 23 & 6.6 & {$[4.4,9.7]$} & 52 & 8.3 & {$[6.4,10.8]$} \\
\hline Para emborracharme o sentirme mejor & 10 & $3.7^{\star}$ & {$[2.0,6.7]$} & 20 & $5.7^{*}$ & {$[3.7,8.7]$} & 30 & 4.8 & {$[3.4,6.8]$} \\
\hline
\end{tabular}


Características distintivas del consumo de alcohol entre hombres y mujeres (continuación)

\begin{tabular}{|c|c|c|c|c|c|c|c|c|c|}
\hline \multirow[b]{2}{*}{ Variables } & \multicolumn{3}{|c|}{ Hombre } & \multicolumn{3}{|c|}{ Mujer } & \multicolumn{3}{|c|}{ Total } \\
\hline & $n$ & $\%$ & IC 95\% & $n$ & $\%$ & IC 95\% & $n$ & $\%$ & IC 95\% \\
\hline \multicolumn{10}{|l|}{ Número de copas que toma } \\
\hline Una a dos copas & 102 & $37.1^{\star \star}$ & {$[31.6,43.0]$} & 188 & $53.4^{\star *}$ & {$[48.2,58.6]$} & 290 & 46.3 & {$[42.4,50.2]$} \\
\hline Tres o más & 128 & 46.5 & {$[40.7,52.5]$} & 142 & 40.3 & {$[35.3,45.6]$} & 270 & 43.1 & {$[39.2,47.0]$} \\
\hline Hasta emborracharme & 45 & $16.4^{\star *}$ & {$[12.4,21.2]$} & 22 & $6.3^{* *}$ & {$[4.1,9.3]$} & 67 & 10.7 & {$[8.5,13.4]$} \\
\hline \multicolumn{10}{|l|}{ Con quién toma } \\
\hline Padres, hermanos o familiares & 80 & $29.1^{\star *}$ & {$[24.0,34.8]$} & 159 & $45.2^{\star \star}$ & {$[40.0,50.4]$} & 239 & 38.1 & {$[34.4,42.0]$} \\
\hline Amigos & 169 & $61.5^{\text {** }}$ & {$[55.5,67.1]$} & 172 & $48.9^{* *}$ & {$[43.6,54.1]$} & 341 & 54.4 & {$[50.5,58.3]$} \\
\hline Solo & 26 & 9.5 & {$[6.5,13.6]$} & 21 & 6.0 & {$[3.9,9.0]$} & 47 & 7.5 & {$[5.7,9.8]$} \\
\hline \multicolumn{10}{|l|}{ Cuál es el efecto mayor por el alcohol } \\
\hline Me sentí relajado, cómodo & 126 & 45.8 & {$[40.0,51.8]$} & 169 & 48.0 & {$[42.8,53.3]$} & 295 & 47 & {$[43.2,51.0]$} \\
\hline Me sentí borracho & 117 & 42.5 & {$[36.8,48.5]$} & 156 & 44.3 & {$[39.2,49.6]$} & 273 & 43.5 & {$[39.7,47.5]$} \\
\hline $\begin{array}{l}\text { Enfermo, me desmayé, al día siguiente } \\
\text { no me acordaba de lo que había pasado }\end{array}$ & 32 & 11.6 & {$[8.3,16.0]$} & 27 & 7.7 & {$[5.3,11.0]$} & 59 & 9.4 & {$[7.4,12.0]$} \\
\hline \multicolumn{10}{|l|}{ Cómo te ven otros cuando tomas } \\
\hline Como alguien normal & 222 & 80.7 & {$[75.6,85.0]$} & 290 & 82.4 & {$[78.0,86.0]$} & 512 & 81.7 & {$[78.4,84.5]$} \\
\hline $\begin{array}{l}\text { Cuando tomo tiendo a ser poco } \\
\text { considerado }\end{array}$ & 12 & 4.4 & {$[2.5,7.6]$} & 19 & 5.4 & {$[3.5,8.3]$} & 31 & 4.9 & {$[3.5,7.0]$} \\
\hline $\begin{array}{l}\text { Mi familia o amigos me aconsejan que } \\
\text { controle o reduzca mi forma de beber }\end{array}$ & 29 & 10.5 & {$[7.4,14.8]$} & 38 & 10.8 & {$[7.9,14.5]$} & 67 & 10.7 & {$[8.5,13.4]$} \\
\hline $\begin{array}{l}\text { Mi familia y amigos me han buscado } \\
\text { ayuda }\end{array}$ & 12 & 4.4 & {$[2.5,7.6]$} & 5 & 1.4 & {$[0.6,3.4]$} & 17 & 2.7 & {$[1.7,4.3]$} \\
\hline \multicolumn{10}{|c|}{ Cómo te sientes acerca de tu forma de beber alcohol } \\
\hline No tengo problema & 129 & 46.9 & {$[41.0,52.9]$} & 192 & 54.5 & {$[49.3,59.7]$} & 321 & 51.2 & {$[47.3,55.1]$} \\
\hline Puedo controlarme & 124 & 45.1 & {$[39.3,51.1]$} & 143 & 40.6 & {$[35.6,45.9]$} & 267 & 42.6 & {$[38.8,46.5]$} \\
\hline $\begin{array}{l}\text { Siento que me puedo controlar pero mis } \\
\text { amigos influyen en mí fácilmente }\end{array}$ & 14 & 5.1 & {$[3.0,8.4]$} & 7 & 2.0 & {$[0.9,4.1]$} & 21 & 3.3 & {$[2.2,5.1]$} \\
\hline $\begin{array}{l}\text { Frecuentemente me siento mal acerca } \\
\text { de mi forma de beber o necesito ayuda }\end{array}$ & 8 & 2.9 & {$[1.5,5.7]$} & 10 & 2.8 & {$[1.5,5.2]$} & 18 & 2.9 & {$[1.8,4.5]$} \\
\hline
\end{tabular}

*Diferencia estadística entre categorías de las variables, al interior de cada sexo

**Diferencia estadística entre hombres y mujeres 
La Tabla 3 muestra la relación entre la experimentación de eventos violentos vividos, la presencia de sintomatología y el consumo de alcohol alguna vez en la vida. Se observan diferencias significativas entre las mujeres que dijeron haber vivido un EVPT y las que no, siendo más frecuente el consumo de alcohol en las primeras (77.4\%, IC = 72.9, 81.3). En hombres, el consumo resultó similar entre quienes vivieron un evento y quienes no; asimismo, el número de hombres y mujeres que dijeron haber vivido un evento de esta naturaleza también fue similar; así como el de quienes eligieron un evento con repercusión actual y los que no.

Por tipo de evento elegido, se observó una mayor frecuencia de consumo de alcohol entre los hombres expuestos a violencia organizada (87.3\%, IC = 80.2, 92.1) que los que no $(67.9 \%, I C=60.4,74.5)$. Asimismo, el uso de esta sustancia fue mayor en las mujeres que reportaron eventos de violencia interpersonal (83.4\%, $I C=77.43,88.1)$, que en quienes no eligieron este tipo de evento $(71.4 \%, I C=64.5,77.35)$.

$81 \%$ de los hombres que dijeron tener síntomas de evitación de tipo embotamiento emocional reportaron haber consumido alcohol, mientras que $79.7 \%$ de las mujeres con esta sintomatología también lo manifestaron; las diferencias encontradas no fueron estadísticamente significativas. En la población total, quienes manifestaron síntomas de evitación, de tipo alejamiento, reportaron significativamente un consumo mayor de alcohol (80.6\%, IC $=76.7,84.0)$, en comparación con quienes no presentaron síntomas $(67.4 \%, I C=56.8,76.5)$.

\section{DISCUSIÓN Y CONCLUSIONES}

Como se observó, cuatro de cada cinco participantes en este estudio habían padecido al menos un EVPT en la vida, de ellos una proporción similar evaluó subjetivamente que uno de estos eventos les seguía afectando hasta la actualidad, lo que da cuenta del impacto que tienen estos sucesos en el bienestar y la salud mental de los y las jóvenes. Kessler et al. (2010) señalan que experiencias como el abuso físico y sexual en la infancia, la exposición a la violencia doméstica y el abuso emocional, psicológico o verbal, incrementan el riesgo de sufrir trastornos del estado de ánimo, de ansiedad, del comportamiento y trastornos del uso de sustancias a lo largo de la vida. Además, Alisic et al. (2014), a partir de una revisión sistemática, reportaron que 15.9\% de niños, niñas y adolescentes desarrollaron TEPT tras un suceso traumático.

Los estudiantes participantes manifestaron vivir el fenómeno de polivictimización, al reportar EVPT de diversos tipos, incluyendo "otras amenazas a la vida" -casi tres cuartas partes-, "experiencias traumáticas en red", que se refieren más a exposiciones indirectas -casi $60 \%$ - y algún tipo de "violencia interpersonal" -la mitad-. El impacto de múltiples EVPT también se observó en la Encuesta Mexicana de Salud Mental en Adolescentes, en la cual las situaciones adversas crónicas vividas durante la infancia más reportadas fueron las relacionadas con el ámbito familiar (violencia, alta exposición a abusos de distinta índole y ser testigos de violencia familiar). Su interrelación con otros factores contextuales, se asoció con distintas enfermedades, incluyendo sintomatología del TEPT y tentativa suicida (Benjet et al., 2009; Borges et al., 2008; Orozco et al., 2008).

Eventos comúnmente relacionados con el TEPT, como la guerra y el abuso sexual, estuvieron presentes en esta población; una tercera parte había estado expuesta o había participado en situaciones de "violencia organizada" y $6 \%$ reportó alguna forma de violencia sexual.

En el Estado de México se están presentando, lo que Médecins Sans Frontières (2015) desde un enfoque humanitario, denomina "Otras situaciones de violencia", pues se observan múltiples manifestaciones no reconocidas por la legislación internacional, pero que tienen consecuencias igualmente destructivas. La población se está enfrentando a contextos, donde se combinan múltiples formas de violencia, que se manifiestan tanto en el ámbito público como en el privado. En el sur del estado, las violencias se relacionan con enfrentamientos armados por luchas de poder entre narcotraficantes y otros grupos de interés; la información sobre el "Caso Tlatlaya" (Morales, 2017) y la secuencia de hechos en el llamado "Triángulo de las Brechas" (Michel, 2015) son sólo una aproximación de lo que sucede en esta zona.

El municipio Ecatepec de Morelos, aun con su poca extensión territorial, es uno de los más poblados del país; y aunque en 2015 se situó como el noveno municipio nacional más violento (Seguridad, Justicia y Paz, 2016), ya para 2017 no se ubicó entre los primeros 20. Esto no quiere decir que hayan disminuido su índice de violencia (Seguridad, Justicia y Paz, 2018a), sino que en otros municipios se incrementó por la presencia de más eventos asociados con enfrentamientos entre cárteles del narcotráfico. Para el primer bimestre de 2018, Ecatepec se situó como el municipio más violento en los últimos cuatro años en el Estado de México; a nivel nacional, ocupa el segundo lugar en delitos de robo con violencia (Seguridad, Justicia y Paz, 2018b). No obstante, siguen siendo escasos los estudios que abordan el impacto de esos sucesos en la salud mental de la población.

La persona expuesta directa o indirectamente a la muerte, a un peligro de daño grave o a una violación sexual, o que percibe una amenaza a su integridad física y psicológica puede presentar síntomas subclínicos del 
Tabla 3

Relación de eventos y sintomatología con el consumo de alcohol entre hombres y mujeres

\begin{tabular}{|c|c|c|c|c|c|c|c|c|c|}
\hline \multirow[b]{3}{*}{ Variables } & \multicolumn{9}{|c|}{ Ha tomado alcohol alguna vez en su vida } \\
\hline & \multicolumn{3}{|c|}{ Hombre } & \multicolumn{3}{|c|}{ Mujer } & \multicolumn{3}{|c|}{ Total } \\
\hline & $n$ & $\%$ & IC 95\% & $n$ & $\%$ & IC 95\% & $n$ & $\%$ & IC 95\% \\
\hline \multicolumn{10}{|c|}{ Vivió algún evento } \\
\hline No & 51 & 70.8 & {$[59.1,80.3]$} & 54 & $55.1^{*}$ & {$[45.0,64.8]$} & 105 & $61.8^{*}$ & {$[54.2,68.8]$} \\
\hline Sí & 224 & 76.2 & {$[71.0,80.7]$} & 298 & $77.4^{*}$ & {$[72.9,81.3]$} & 522 & $76.9^{*}$ & {$[73.5,79.9]$} \\
\hline \multicolumn{10}{|c|}{ Exposición y participación en la violencia organizada } \\
\hline No & 114 & $67.9^{*}$ & {$[60.4,74.5]$} & 191 & 74.3 & {$[68.6,79.3]$} & 305 & $71.8^{*}$ & {$[67.3,75.9]$} \\
\hline Sí & 110 & $87.3^{*}$ & {$[80.2,92.1]$} & 107 & 83.6 & {$[76.0,89.1]$} & 217 & $85.4^{*}$ & {$[80.5,89.3]$} \\
\hline \multicolumn{10}{|c|}{ Violencia interpersonal } \\
\hline No & 97 & 74.6 & {$[66.3,81.4]$} & 137 & $71.4^{*}$ & {$[64.5,77.35]$} & 234 & 72.7 & {$[67.5,77.3]$} \\
\hline Sí & 127 & 77.4 & {$[70.3,83.2]$} & 161 & $83.4^{*}$ & {$[77.43,88.1]$} & 288 & 80.7 & {$[76.2,84.5]$} \\
\hline \multicolumn{10}{|c|}{ Violencia sexual } \\
\hline No & 219 & 76.3 & {$[71.0,80.9]$} & 269 & 76.2 & {$[71.5,80.4]$} & 488 & 76.3 & {$[72.8,79.4]$} \\
\hline Sí & 5 & 71.4 & {$[21.5,95.8]$} & 29 & 90.6 & {$[73.3,97.1]$} & 34 & 87.2 & {$[71.8,94.8]$} \\
\hline \multicolumn{10}{|c|}{ Otras amenazas para la vida } \\
\hline No & 41 & 73.2 & {$[59.8,83.4]$} & 97 & 80.8 & {$[72.7,87.0]$} & 138 & 78.4 & {$[71.6,83.9]$} \\
\hline Sí & 183 & 76.9 & {$[71.1,81.8]$} & 201 & 75.8 & {$[70.3,80.7]$} & 384 & 76.3 & {$[72.4,79.9]$} \\
\hline \multicolumn{10}{|c|}{ Experiencias traumáticas en red } \\
\hline No & 109 & 74.1 & {$[66.4,80.6]$} & 89 & 71.8 & {$[63.1,79.1]$} & 198 & 73.1 & {$[67.4,78.0]$} \\
\hline Sí & 115 & 78.2 & {$[70.7,84.2]$} & 209 & 80.1 & {$[74.8,84.5]$} & 324 & 79.4 & {$[75.2,83.1]$} \\
\hline \multicolumn{10}{|c|}{ Consideraron un evento con repercusión actual } \\
\hline No & 41 & 67.2 & {$[54.2,78.0]$} & 55 & 73.3 & {$[62.0,82.3]$} & 96 & 70.6 & {$[62.3,77.7]$} \\
\hline Sí & 183 & 78.5 & {$[72.8,83.4]$} & 243 & 78.4 & {$[73.4,82.6]$} & 426 & 78.5 & {$[74.8,81.7]$} \\
\hline \multicolumn{10}{|c|}{ Síntomas de evitación de tipo embotamiento emocional } \\
\hline No & 30 & 68.2 & {$[52.5,80.6]$} & 31 & 70.5 & {$[54.9,82.4]$} & 61 & 69.3 & {$[58.7,78.2]$} \\
\hline Sí & 153 & 81.0 & {$[74.7,86.0]$} & 212 & 79.7 & {$[74.4,84.1]$} & 365 & 80.2 & {$[76.3,83.6]$} \\
\hline \multicolumn{10}{|c|}{ Síntomas de reexperimentación de tipo fisiológica } \\
\hline No & 74 & 74.7 & {$[65.1,82.4]$} & 82 & 73.9 & {$[64.8,81.3]$} & 156 & 74.3 & {$[67.9,79.8]$} \\
\hline Sí & 109 & 81.3 & {$[73.7,87.1]$} & 161 & 80.9 & {$[74.8,85.8]$} & 270 & 81.1 & {$[76.5,85.0]$} \\
\hline \multicolumn{10}{|c|}{ Síntomas de evitación de tipo alejamiento } \\
\hline No & 33 & 68.8 & {$[53.9,80.6]$} & 27 & 65.9 & {$[49.6,79.1]$} & 60 & $67.4^{\star}$ & {$[56.8,76.5]$} \\
\hline Sí & 150 & 81.1 & {$[74.7,86.1]$} & 216 & 80.3 & {$[75.1,84.6]$} & 366 & $80.6^{*}$ & {$[76.7,84.0]$} \\
\hline \multicolumn{10}{|c|}{ Síntomas de hiperactivación } \\
\hline No & 18 & 62.1 & {$[42.4,78.4]$} & 31 & 83.8 & {$[67.4,92.8]$} & 49 & 74.2 & {$[62.1,83.5]$} \\
\hline Sí & 165 & 80.9 & {$[74.8,85.7]$} & 212 & 77.7 & {$[72.3,82.2]$} & 377 & 79 & {$[75.1,82.5]$} \\
\hline \multicolumn{10}{|c|}{ Síntomas de reexperimentación de tipo cognitiva } \\
\hline No & 54 & 78.3 & {$[66.7,86.6]$} & 51 & 79.7 & {$[67.7,88.0]$} & 105 & 78.9 & {$[71.1,85.1]$} \\
\hline Sí & 129 & 78.7 & {$[71.6,84.3]$} & 192 & 78.0 & {$[72.4,82.8]$} & 321 & 78.3 & {$[74.0,82.0]$} \\
\hline
\end{tabular}

Notas:

a) De los 627 estudiantes que dijeron consumir alcohol, 522 vivieron al menos un evento de vida de distinto tipo y 426 manifestaron repercusión actual con variación en el factor de sintomatología.

*Diferencia estadística entre categorías de las variables, al interior de cada sexo. 
TEPT, al haber vivenciado un evento con las características del Criterio A del Manual Diagnóstico y Estadístico de Trastornos Mentales (DSM-5); este hecho se convierte en el factor inicial de su diagnóstico (APA, 2013). Por ello, no fue sorpresivo que se observaran porcentajes elevados de sintomatología postraumática: presencia de al menos un síntoma en más de $80 \%$ de los participantes en las dimensiones de evitación -tanto emocional como conductual-, evaluadas de manera independiente, así como de hiperactivación fisiológica. Asimismo, entre 60 y $70 \%$ reportó reexperimentación fisiológica y cognitiva. Es decir que aunque no se puede hablar de un diagnóstico de TEPT, se observa una importante afectación en estos jóvenes, manifestada, principalmente, en el intento de evitar sentir o hacer actividades que se asocian con el evento experimentado; los afectados presentan, también, síntomas como insomnio, estallidos de ira e hipervigilancia.

En cuanto al consumo de alcohol, siete de cada 10 participantes reportaron haberlo hecho alguna vez, mientras que en la Encuesta Nacional de Consumo de Drogas en Estudiantes (ENCODE, 2014) se encontró que alrededor de la mitad de los estudiantes de secundaria y bachillerato había ingerido esta sustancia alguna vez en su vida (53.2\%): $54 \%$ de los hombres y $52.5 \%$ de las mujeres (Villatoro et al., 2016).

No se encontraron diferencias significativas en los motivos que los hombres y mujeres señalaron para comenzar a beber alcohol -entre los que destaca "su sabor"-, ni en el hecho de que consumir haya respondido mayoritariamente a la curiosidad, ni en la edad de inicio -entre los 10 y 15 años-, lo que coincide con lo reportado en la ENCODE 2014, en la que el promedio de edad de inicio fue de 12.6 años (Villatoro et al., 2016).

Los resultados muestran un patrón de consumo muy similar en ambos sexos, en la medida en que las mujeres han ido incrementando su consumo de alcohol, posiblemente por cambios culturales que han llevado a que las personas más jóvenes, en general, y las mujeres, en particular, acepten cada vez más los comportamientos de consumo social. Lo cual coincide con lo reportado en la Encuesta Nacional de Consumo de Drogas, Alcohol y Tabaco (ENCODAT, 2016-2017) en población adolescente (12 a 17 años), que indica que 39.8\% había consumido alcohol alguna vez en la vida $(41.7 \%$ hombres y $37.9 \%$ mujeres) y $28 \%$ en el último año (28.8\% hombres y $27.2 \%$ mujeres; Villatoro-Velásquez, 2017).

Entre los participantes se encontró que los hombres siguen consumiendo más alcohol cuando beben: casi tres veces más hombres que mujeres beben hasta emborracharse. Ello coincide con lo reportado previamente: la ENCODE (2014) señaló que beber cinco copas o más en una sola ocasión en el último mes se presentó en
$14.5 \%$ de los estudiantes (15.7\% hombres, $13.3 \%$ mujeres), mientras que para Ecatepec fue de 16\% (16.4\% en hombres y $15.6 \%$ en mujeres; Villatoro et al., 2016); la ENCODAT (2016-2017) resaltó que $8.9 \%$ de los hombres y $7.7 \%$ de las mujeres bebían excesivamente (Villatoro-Velásquez et al. 2017).

Aunque poco más de la mitad de los estudiantes que participaron en esta investigación dijo no tener problemas con su consumo de alcohol, destacó que entre 8 y $12 \%$ de los que han bebido, se han sentido enfermos, se desmayaron o al día siguiente no se acordaban de lo que había pasado; una proporción similar reportó que su familia o amigos les aconsejó que controle o reduzca su consumo. No hubo diferencias significativas entre sexos en este rubro, de modo que se habla de que uno de cada 10 estudiantes presenta ya problemas asociados con el consumo y requiere de una intervención específica para no desarrollar una dependencia de la sustancia. La ENCODAT (2016-2017) previamente había enfatizado la existencia de la dependencia del alcohol en $2.2 \%$ de la población total de entre 12 y 65 años de edad: 6.5 veces mayor en los hombres (3.9\%) que en las mujeres $(0.6 \%)$, por ello, es de suma importancia atender el consumo actual de los adolescentes, así como las variables que influyen en dicho consumo, como la forma de obtener el alcohol: las mujeres consiguen las bebidas alcohólicas principalmente de sus padres, hermanos $u$ otros familiares, y toman alcohol con ellos en mayor medida que los hombres, quienes consumen más con sus amigos (Villatoro-Velasquez et al. 2017).

En cuanto al impacto subjetivo del evento hasta la época actual, sí hubo diferencias tanto en hombres como en mujeres, respecto al consumo de alcohol entre los estudiantes que vivieron algún tipo de EVPT y los que no, lo cual concuerda con estudios como el de Forman- Hoffman et al. (2016), quienes en Estados Unidos encontraron mayor consumo de drogas ilegales, en adultos con exposición a uno o más EVPT en su vida, en comparación con quienes no tuvieron dicha exposición.

Según el tipo de evento, los hombres con exposición y participación en la violencia organizada dijeron consumir más alcohol que quienes no lo vivieron. En las mujeres, la misma diferencia se encontró entre quienes habían vivido violencia interpersonal y las que no la habían experimentado. Vargas, del Castillo \& Guzmán (2016) encontraron una relación positiva entre el nivel de estrés y el consumo de alcohol en estudiantes mexicanos; los autores atribuyeron este hecho a que el consumo de alcohol reduce la respuesta fisiológica al estrés, si la persona presenta escasas habilidades para controlar la situación estresante o para manejar sus propias emociones y buscar apoyo social. 
Los resultados refieren que la adversidad y la violencia se asocian con el consumo de alcohol de hombres y mujeres; resalta que si no se padecen estos eventos, persiste una diferencia importante que pone a los hombres en una situación de mayor consumo. Por lo anterior, es importante reflexionar en la importancia del género para comprender dichos resultados, así como considerar las dimensiones genéticas, fisiológicas, psicosociales y ambientales asociadas con el uso y abuso de drogas, en específico, del alcohol. Los tratamientos y la prevención deben adaptarse a las diferentes necesidades de hombres y mujeres, y considerar su etapa de vida (National Institute on Drug Abuse, NIDA, 2002).

Los hallazgos de este trabajo están sujetos a limitaciones: 1) el diseño transversal retrospectivo puede ocasionar sesgos de recuerdo en los estudiantes, lo que implica sobrerrepresentar los EVPT más cercanos en el tiempo o de mayor impacto, impedir el estudio de las trayectorias de evolución entre un EVPT y la aparición (o desaparición) de síntomas de TEPT, y evitar la realización de inferencias causales sobre el papel de los factores estudiados; 2) el uso de un instrumento de cribado permite hablar solamente de sintomatología, aunque se utilicen cinco dimensiones; 3) sólo se usaron reactivos de manera independiente en el cuestionario para evaluar el consumo de alcohol, las características psicométricas del instrumento requieren ser analizadas; 4) solamente se llevaron a cabo análisis bivariados sin controlar otras variables; y 5) la muestra por conveniencia no permite generalizar los resultados a todos los estudiantes mexiquenses. Futuras investigaciones en la población estudiada podrían considerar investigaciones con diseños en sentido bidireccional que permitan establecer relación de causalidad entre las variables.

\section{FUENTES DE FINANCIAMIENTO}

La presente investigación formó parte de un proyecto de doctorado en Ciencias de la Salud (2014-2017) en la Universidad Autónoma del Estado de México, con registro en el Padrón Nacional de Posgrados de Calidad del Consejo Nacional de Ciencia y Tecnología (CONACYT). Las opiniones, los hallazgos y las conclusiones aquí presentadas son de las autoras y no necesariamente reflejan las del CONACYT.

\section{CONFLICTOS DE INTERÉS}

Las autoras declaramos que no hay conflicto de interés en los datos presentados.

\section{AGRADECIMIENTOS}

Al personal del Centro de Investigación en Ciencias Médicas de la Universidad Autónoma del Estado de México y a las escuelas participantes en el estudio, por las facilidades otorgadas. A los integrantes del Grupo de Investigación de Violencia y Género, del Instituto Nacional de Psiquiatría Ramón de la Fuente Muñiz, por las aportaciones al proyecto.

\section{REFERENCIAS}

Alisic, E., Zalta, A. K., Van Wesel, F., Larsen, S. E., Hafstad, G. S., Hassanpour, K., ... Smid, G. E. (2014). Rates of post-traumatic stress disorder in trauma-exposed children and adolescents: meta-analysis. The British Journal of Psychiatry, 204(5), 335340.

American Psychiatric Association. (2013). Section II: disgnostic criteria and codes: trauma and stressor related disorders. En APA. Disgnostic and Statisticial Manual of Mental Disorders. Arlington: Autor.

Benjet, C., Borges, G., Medina-Mora, M. E., Zambrano, J., Cruz, C., \& Méndez, E. (2009). Descriptive epidemiology of chronic childhood adversity in mexican adolescents. Journal of Adolescent Health, 45(5), 483-489. doi: 10.1016/j.jadohealth.2009.03.002

Borges, G., Benjet, C., Medina-Mora, M. E., Orozco, R., Molnar, B. E., \& Nock, M.K. (2008). Traumatic events and suicide-related outcomes among Mexico City adolescents. Journal of Child Psychology and Psychiatry, 49(6), 654-666. doi: 10.1111/j.14697610.2007.01868.x.

Cabildo, M. (26 de octubre, 2011). Violencia deja 12 muertos en cinco estados. Proceso. Recuperado de http://www.proceso.com. mx/286196/violencia-deja-12-muertos-en-cinco-estados

Cyr, K., Chamberland, C., Clement, M. E., Wemmers, J. A., CoIlin-Vézina, D., Lessard, G., ... Damant, D. (2017). The impact of lifetime victimization and polyvictimization on adolescents in Québec: mental health symptoms and gender differences. Violence and Victims, 32(1), 3-21. doi: 10.1891/0886-6708.VV-D-1400020.

Fairbrook, S. W. (2013). The physical and mental health effects of community violence exposure in pre-adolescent and adolescent youth. Journal of Student Nursing Research, 6(1), 24-30.

Ferri, P. (2014). Exclusiva: testigo revela ejecuciones en el Estado de México. Esquire Latinoamérica. Recuperado de http://www. esquirelat.com/reportajes/exclusiva-testigo-revela-ejecucionesen-el-estado-de-mexico/

Forman-Hoffman, V. L., Bose, J., Batts, K. R., Glasheen, C., Hirsch, E., Karg, R. S., ... Hedden, S. L. (2016). Correlates of lifetime exposure to one or more potentially traumatic events and subsequent posttraumatic stress among. En Substance Abuse and Mental Health Services Administration. (2016). CBHSQ Data Review. Rockville: Autor. 
Gómez Hernández, H. L. \& Gómez-Maqueo, E. L. (2016). Incidencia de exposición a la violencia en la comunidad en estudiantes adolescentes de la Ciudad de México. Psicología y Salud, 26(2), 217-224.

Hill, S. Y., Shen, S., Lowers, L., \& Locke, J. (2000). Factors predicting the onset of adolescent drinking in families at high risk for developing alcoholism. Biological Psychiatry, 48(4), 265-275.

Hughes, K., Bellis, M. A., Hardcastle, K. A., Sethi, D., Butchart, A., Mikton, C., ... Dunne, M. P. (2017). The effect of multiple adverse childhood experiences on health: a systematic review and meta-analysis. The Lancet Public Health, 2(8), e356-e366. doi: 10.1016/S2468-2667(17)30118-4

Instituto Nacional de Estadística y Geografía. (2015). Encuesta de Cohesión Social para la Prevención de la Violencia y la Delincuencia (ECOPRED) 2014. Principales resultados. México: Autor. Recuperado de http://internet.contenidos.inegi.org.mx/contenidos/ productos/prod_serv/contenidos/espanol/bvinegi/productos/ nueva_estruc/promo/ecopred14_presentacion_ejecutiva.pdf

Instituto Nacional para el Federalismo y Desarrollo Municipal. (2017). Enciclopedia de los municipios y delegaciones de México, Estado de México. Recuperado de http://www.inafed.gob. mx/work/enciclopedia/EMM15mexico/index.html

Jiménez, E. I. (2004). Expectativas y abuso de alcohol en adolescentes. (Tesis de maestría). Monterrey: Universidad Autónoma de Nuevo León. Recuperado de http://cd.dgb.uanl.mx.

Kaysen, D., Dillworth, T. M., Simpson, T., Waldrop, A., Larimer, M. E., \& Resick, P. A. (2007). Domestic violence and alcohol use: trauma-related symptoms and motives for drinking. Addictive Behaviors, 32(6), 1272-1283. doi: 10.1016/j.addbeh.2006.09.007

Kessler, R. C., McLaughlin, K. A., Green, J. G., Gruber, M. J., Sampson, N. A., Zaslavsky, A. M., ... Benjet, C. (2010). Childhood adversities and adult psychopathology in the WHO World Mental Health Surveys. The British Journal of Psychiatry, 197(5), 378385. doi: 10.1192/bjp.bp.110.080499

Kilpatrick, D. G., Acierno, R., Saunders, B., Resnick, H. S., Best, C. L., \& Schnurr, P. P. (2000). Risk factors for adolescent substance abuse and dependence: data from a national sample. Journal of Consulting and Clinical Psychology, 68(1), 19-30.

Löfving-Gupta, S., Willebrand, M., Koposov, R., Blatný, M., Hrdlička, M., Schwab-Stone, M., ... Ruchkin, V. (2017). Community violence exposure and substance use: cross-cultural and gender perspectives. European Child \& Adolescent Psychiatry, 27(4), 493-500. doi: 10.1007/s00787-017-1097-5

Médecins Sans Frontières. (2015). OSV, towards a new paradigm of violence for MSF operations. Analytical report for operations. (Documento no publicado). Brasil: Brazilian Medical Unit.

Medina-Mora, M., Borges-Guimaraes, G., Lara, C., Ramos-Lira, L., Zambrano, J., \& Fleiz-Bautista, C. (2005). Prevalencia de sucesos violentos y de trastorno por estrés postraumático en la población mexicana. Salud Pública de México, 47(1), 8-21.

Mels, C., \& Fernández, L. (2015). Violencia comunitaria en adolescentes desfavorecidos: exposición, impacto percibido y conse- cuencias psicológicas. Revista de Psicología, 24(1), 1-21. doi: 10.5354/0719-0581.2015.36863.

Mendoza-Mojica, S. A., Márquez-Mendoza, O., Veytia-López, M. Ramos-Lira, L. E., \& Orozco-Zavala, R. (2017). Eventos potencialmente traumáticos y sintomatología postraumática en estudiantes de preparatoria. Salud Pública de México, 59(6), 665674. doi: 10.21149/8716.

Mendoza-Mojica, S. A., Márquez, O., Guadarrama, R., \& Ramos, L. (2013). Medición del Trastorno por Estres Postraumático (TEPT) en universitarios mexicanos. Salud Mental, 36(6), 493-503.

Michel, E. (8 de agosto, 2015). Piden 172 reporteros protección al gobierno. El Universal. Recuperado de http://www.eluniversal. com.mx/articulo/periodismo-de-investigacion/2015/08/8/piden172-reporteros-proteccion-al-gobierno.

Morales, A. (20 de febrero, 2017). Ordenan a CNDH dar versión del caso Tlatlaya. El Universal. Recuperado de http://www.eluniversal. com.mx/articulo/nacion/seguridad/2017/02/20/ordenan-cndhdar-version-del-caso-tlatlaya

National Institute on Drug Abuse. (2002). In drug abuse, gender matters. Recuperado de https://archives.drugabuse.gov/newsevents/nida-notes/in-drug-abuse-gender-matters

Organización Mundial de la Salud. (2002). Informe mundial sobre la violencia y la salud. Resumen. Washington, D.C.: Organización Panamericana de la Salud, Oficina Regional para las Américas de la Organización Mundial de la Salud. Recuperado de http:// www.who.int/violence_injury_prevention/violence/world_report/ es/summary_es.pdf

Organización Mundial de la Salud. (2013). Global and regional estimates of violence against women: prevalence and health effects of intimate partner violence and non-partner sexual violence. Suiza: Autor.

Orozco, R., Borges, G., Benjet, C., Medina-Mora, M. E., \& López-Carrillo, L. (2008). Eventos traumáticos de vida y trastorno de estrés postraumático en adolescentes mexicanos: resultados de encuesta. Salud Pública de México, 50(supl.1), s29-s37.

Proceso. (29 de enero, 2011). Estado de México, Mítines en pueblos de narcos. Recuperado de http://www.proceso.com.mx/261710/ estado-de-mexico-mitines-en-pueblos-de-narcos

Proceso. (28 de abril, 2014). Asesinan a mando policiaco y levantan a siete agentes en Luvianos, Estado de México. Recuperado de http://www.proceso.com.mx/370842/ejecutan-a-mando-policiacoy-levantan-a-siete-policias-en-luvianos-estado-de-mexico

Ramos-Lira, L., Saltijeral-Méndez, M. T., Romero-Mendoza, M., Caballero-Gutiérrez, M. A., \& Martínez-Vélez, N. A. (2001). Violencia sexual y problemas asociados en una muestra de usuarias de un centro de salud. Salud Pública de México, 43(3), 182-191.

Seguridad, Justicia y Paz. (2016). La violencia en los municipios de México, 2015. Recuperado de http://www.seguridadjusticiaypaz. org.mx/biblioteca/prensa/send/6-prensa/235-la-violencia-en-losmunicipios-de-mexico-2015

Seguridad, Justicia y Paz. (2018a). Tecomán es el nuevo municipio más violento de México; le siguen Manzanillo y Chilapa 
de Álvarez. Recuperado de https://www.seguridadjusticiaypaz. org.mx/sala-de-prensa/1515-tecoman-es-de-nuevo-el-municipiomas-violento-de-mexico-le-siguen-manzanillo-y-chilapa-dealvarez.

Seguridad, Justicia y Paz. (2018b). Crecen muertes violentas en el Estado de México y Puebla. Recuperado de https://www. seguridadjusticiaypaz.org.mx/seguridad/1523-crecen-muertesviolentas-en-el-estado-de-mexico-y-puebla.

Shorey, R. C., Rhatigan, D. L., Fite, P. J., \& Stuart, G. L. (2011). Dating violence victimization and alcohol problems: an examination of the stress-buffering hypothesis for perceived support. Partner Abuse, 2(1), 31-45. doi: 10.1177/1557988311429194.

Teicher, M. H., \& Samson, J. A. (2016). Annual research review: enduring neurobiological effects of childhood abuse and neglect. Journal of Child Psychology and Psychiatry, 57(3), 241-266.
Vargas, R., del Castillo, A., \& Guzmán, R. M. E. (2016). Estrés, depresión y consumo de alcohol en estudiantes de bachillerato de México: diferencias por sexo y escuela. European Scientific Journal, 12(32), 94-109.

Villatoro, J. A., Medina-Mora, M. E., Del Campo, R. M., Fregoso, D. A., Bustos, M. N., Reséndiz, E., ... Cañas, V. (2016). El consumo de drogas en estudiantes de México: tendencias y magnitud del problema. Salud Mental, 39(4), 193-203.

Villatoro-Velásquez, J. A., Reséndiz-Escobar, E., Mujica-Salazar, A., Bretón-Cirett, M., Cañas-Martínez, V., Soto-Hernández, I., ... Mendoza-Alvarado (2017). Encuesta Nacional de Consumo de Drogas, Alcohol y Tabaco 2016-2017: Reporte de Alcohol. (ENCODAT, 2016-2017). México: Instituto Nacional de Psiquiatría Ramón de la Fuente Muñíz, Instituto Nacional de Salud Pública, Instituto Nacional Contra las Adicciones, Secretaría de Salud. 